\title{
A Simulation-Based Workflow to Assess Human-Centric Daylight Performance
}

\author{
Siobhan Rockcastle, María Lovísa Ámundadóttir, and Marilyne Andersen \\ LIPID, ENAC, EPFL \\ Lausanne, Switzerland \\ Siobhan.rockcastle@epfl.ch
}

\begin{abstract}
This paper will present an annual simulation-based workflow for assessing human perceptual and non-visual responses to daylight across a series of view positions in an architectural case study. Through the integration of mathematical models used to predict visual interest and non-visual health potential, this paper will introduce an automated workflow to assess an array of view positions (located at eye level) under varied sky conditions and across multiple view directions to analyze the predicted impacts of daylight on perception and health in architecture. This approach allows for a spatial and occupant centric analysis of daylight using an integrated simulation-based approach.
\end{abstract}

\section{Author Keywords}

Daylight perception, non-visual health potential, humancentric performance, visual interest, non-visual response, daylight performance, lighting simulation.

\section{INTRODUCTION}

Daylight is a powerful element in the experience, vitality, and expression of architecture. Its intensity, direction, and color can transform the visual and physiological responses of an occupant, but the inherently variable nature of these elements and their impact on performance can make a holistic assessment particularly challenging. An architect must integrate, control, and re-distribute daylight to meet general illumination requirements, while fulfilling aesthetic design objectives and creating a comfortable and healthy occupant experience. This task is made even more complex by the temporal variability of solar altitude and sky conditions. Most architects and building engineers are familiar with general illumination requirements, but the perceptual and health-related impacts of daylight are less frequently integrated alongside more traditional performance targets in daylight design. A brief overview of research in daylight perception and non-visual effects of light will help position the performance modules in this paper and are further detailed in Section 2.2.

\subsection{Impacts of Daylight on Perception}

Characteristics of daylight such as composition and contrast can create strong impacts on our perceptual experience in architecture. While many architects openly acknowledge this potential, research into objectifying and/or quantifying these characteristics has been limited [1]. Past studies have revealed a link between daylight and occupant impressions of pleasantness and/or interest using indicators such as average brightness, luminous distribution, and luminance diversity [2-6]. These studies have generally found that some amount of brightness and luminous diversity creates a positive impact on impressions of interest and pleasantness, while excessive brightness and diversity can cause visual discomfort. Although widespread consensus on the measurable impacts of brightness, distribution and diversity has yet to be reached, there are a number of recent studies which have proposed methods of quantifying the compositional characteristics of daylight and their impacts on perception. The Luminance Difference Index [7] is a physically based measure which quantifies the compositional diversity of luminance levels across a range of view directions. The authors of this study found a link between higher luminance diversity and increased ratings of pleasantness, but a dependence on physical measurements limits the use of this index. Integration within the design phase necessitates a simulation-based method capable of assessing unbuilt proposals.

A set of algorithms proposed by Rockcastle et al. [1] were developed to assess rendered images and quantify the compositional and temporal variability of daylight across a fixed view position. Further development of these algorithms by Rockcastle et al. [8] used an online survey to collect subjective ratings for a series of attributes in daylit renderings and fit the distribution of responses for calming exciting to a modified algorithm called mSC (modified spatial contrast). The model generated from this algorithm and survey will be introduced as a performance module in Section 2.2 to predict instances of visual interest from rendered scenes.

\subsection{Impacts of Light on Non-visual Responses}

Shifting from visual to non-visual effects of light, there are several factors that have been linked with health-related performance indicators, such as the quantity of light we receive over time and the duration/ timing of that exposure. Increased daytime exposure to bright light (<1000 lx) has been positively associated with sleep quality [9] and shown beneficial effects on alertness and vitality [10-11] Light exposure does not always induce positive effects, however, as night-time exposure of dim light (>100 lx) can shift the 
circadian clock and disturb other behavioral/physiological processes such as melatonin production [12]. The concept of 'healthy' lighting is still relatively new in the lighting community, as the existence of novel photoreceptors called ipRGCs was only discovered at the beginning of this century [13-14]. As such, there is a large knowledge gap in understanding how different light properties can influence non-visual responses and how those responses can influence occupant health and wellbeing in buildings.

The estimation of a healthy daily light dose poses many challenges, in part due to the relative novelty of research in this areas, but helping to inform decisions that promote health and wellbeing is beneficial to the lighting design community. Non-visual responses must be evaluated as a dynamic system, which adapt to intensity, wavelength, duration, history, and timing of light received at the eye and ultimately at the retina. Based on recent work by Amundadottir [15], this paper applies a novel model, called non-visual direct-response (nvR $R_{D}$ model). This model evaluates the non-visual health potential of light by integrating its underlying photobiological properties on responses in humans. The function and implementation of the $n v R_{D}$ model will be further explained in Section 2.2.

\subsection{Integrated Approach}

Due to the novelty of mathematical models existing in this area of occupant-centric daylight assessment, we have only begun to explore approaches to a computational evaluation. A recent study by Amundadottir et al. simulated a time series of HDR renderings to assess multiple view directions for dynamic impacts of non-visual health potential, visual interest, and gaze behavior [16]. While this approach laid the groundwork for assessing dynamic changes in vertical illuminance and luminance from a human view point, it was limited to a single, fixed view position. As humans generally move through space, a range of view positions would be required to evaluate the diversity of daylight conditions experienced throughout an architectural space.

The workflow presented in this paper will evaluate visual interest and non-visual health potential using a platform which integrates performance modules across an array of view positions and view directions. The results will be shown both spatially (across view positions) and temporally (over time for a select view position) in a select architectural case study. To showcase this approach, the authors have chosen to analyze two floors of the Ryerson Student Learning Center by Snøhetta and Zeidler Partnership in Toronto, Canada.

\section{DEVELOPMENT OF AN INTEGRATED PLATFORM}

As stated above, the primary motivation for development of this platform is to integrate a performance assessment of daylight throughout the building at an occupant scale. The platform provides a simple interface to guide users step-bystep through the simulation setup, resulting in an automated workflow to define inputs and generate the scripts required for the lighting simulation. Using Radiance as the background simulation engine, this platform provides the necessary inputs to the $\mathrm{mSC}$ and $\mathrm{nvR}_{\mathrm{D}}$ performance modules introduced in Sections 1.1-1.2 (explained further in Section 2.2). After the simulation results have been analyzed, performance is shown spatially and temporally.

\subsection{Simulation Workflow}

Generalizing the concept of a time-series simulated for a single, centralized position in space [16], this workflow has been implemented to the simulation of multiple viewpoints in space over any number of desired moments and sky conditions. While integrating perceptual and health-based performance modules in a manner that is accessible for less experienced users, the platform remains flexible for more advanced users who want to override defaults. The user is guided through this workflow step-by-step using a simple interface that runs Radiance to generate illuminance and luminance-based outputs.

The simulation workflow is as follows:

1. Load a 3D model (OBJ, DXF, 3DS, SKP) and convert it to RAD material and geometry files. Alternatively, RAD and material files made elsewhere can be loaded directly.

2. Select the site location, weather and moment distribution, and create OCT files (one per moment and sky condition).

3. Define the position of analysis nodes by reading in a text files or export of point locations from a geometry modeling platform (like Rhino).

4. Run the simulation locally (or use the scripts to run simulations on an external server) to generate illuminance and/or luminance outputs.

5. Compute $\mathrm{mSC}$ and $\mathrm{nvR}_{\mathrm{D}}$ performance modules from the obtained illuminance and luminance data.

6. Visualize the results spatially and temporally.

One of the advantages of using this platform is more efficient data management, where results are stored in an organized data folder system and temporal files are deleted when no longer needed. To speed up simulation times, the location of ambient files is shared, which makes the rendering of many view positions possible without a linear increase in rendering time. Using a method of extracting multiple views from a single $360^{\circ}$ image, we store only one rendered scene but from there we can generate as many $180^{\circ}$ fisheye images as requested.

Instead of running the simulation locally on a personal computer, the platform can generate shell scripts for running computationally demanding projects on an external server. In the background, the platform creates a project folder, which must be transferred as a whole to ensure that the platform recognizes the project. In this example, the authors used a multi-core server to run individual jobs in parallel. Figure 1 shows an overview of this process from geometry model to the application of performance modules. 


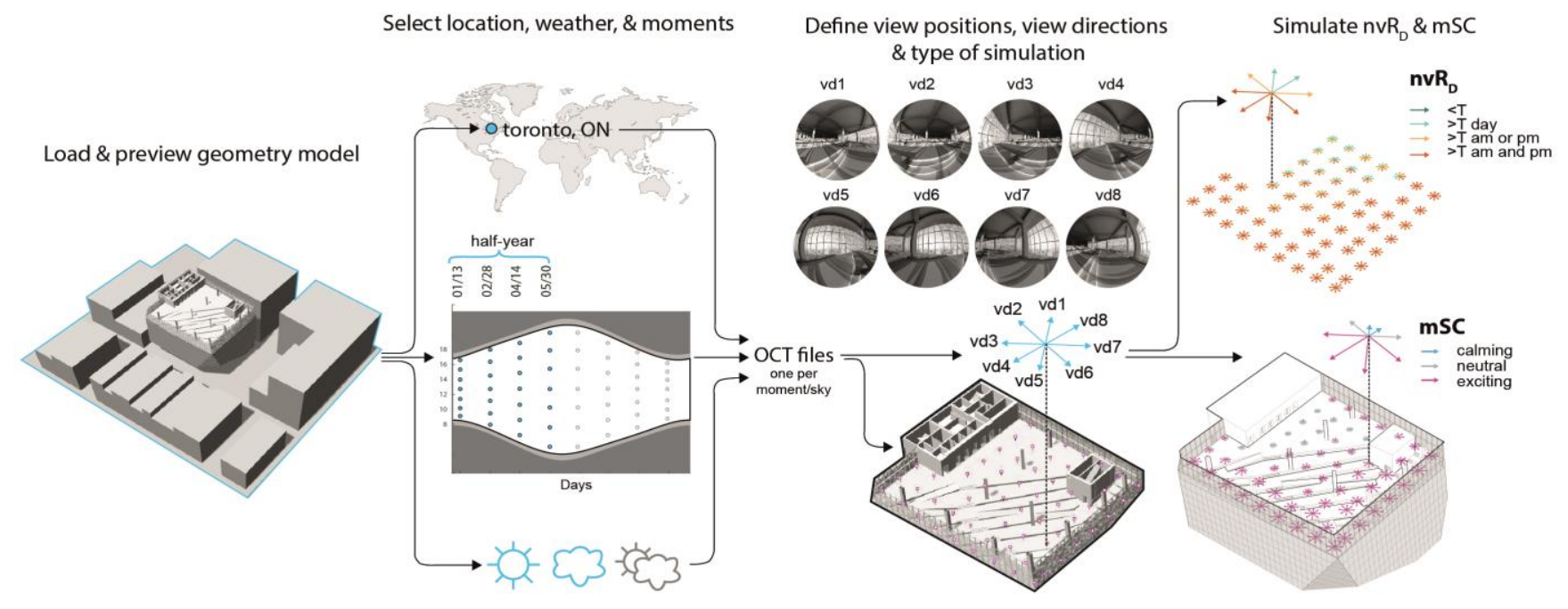

Figure 1. Shows the workflow for our proposed platform; load and preview a geometry models, select location/instances/sky conditions, define location of analysis points/view directions/type of simulation, and run desired performance models $\left(\mathrm{mSC}_{\mathrm{N}} \mathrm{nvR} \mathrm{R}_{\mathrm{D}}\right)$.

\subsection{Performance Modules}

While a detailed mathematical description of the two prediction models described in this paper are introduced elsewhere $[8,15,16]$, the following section will outline the implementation of these models into the proposed simulation platform.

\section{Predicting Visual Interest in Renderings}

The study briefly introduced at the end of Section 1.1, proposed an image-based algorithm called mSC to predict attributes of visual interest in daylight renderings. The $\mathrm{mSC}$ algorithm was modified from a neighborhood measure called RAMMG which was developed to predict contrast perception in digital images [17]. An online experiment with 167 subjects was used to compare a broad range of image-based algorithms to subject ratings of visual interest using 7-point bi-polar ordinal scales from calming exciting and subdued - stimulating. The results of this study found that Pearson Correlation Coefficient values between median ratings of $\mathrm{mSC}$ [8] and calming- exciting and subdued - stimulating were significantly correlated $(r \geq 0.78$, $p<0.001)$ From a population of 167 subjects, logistic regression was used to fit $\mathrm{mSC}$ predictions to the distribution of subject responses for each rendering. The fitted logistic function in [8] computes the probability distribution that subjects would rate a rendering in the calming or exciting spectrum based on image composition. The odds of achieving ratings 1-3 on a 7-point scale are significant at $p<0.05$. More detail can be found in [8].

The mSC algorithm is computed on hemispherical images derived from $360^{\circ}$ fisheye HDR images obtained using rpict > pinterp in Radiance. Each Radiance picture is then tonemapped and compressed using pcond > ra_bmp to provide the appropriate image-based input for computing mSC. Based on the computed mSC value, the fitted model then returns a prediction of visual interest. From the fitted logistic function [8], two thresholds were determined to predict perceptions of calm and excitement (Section 3.4) in a majority percentage of the surveyed population. The application and threshold predictions of calm and excitement mentioned above are described in depth in [16].

\section{Predicting Non-visual Health Potential}

Parallel research in non-visual health (introduced in Section 1.2) proposed a novel model called the non-visual directresponse $\left(n v R_{D}\right)$ model which predicts the relative nonvisual responses to light with the aim of evaluating the dynamic light-response behavior that occurs under realworld settings [16]. The $n v R_{D}$ takes effective irradiance as an input [18]. Currently, the vertical illuminance is computed using rtrace in Radiance and then these values are scaled assuming a constant spectral power distribution of CIE standard indoor illuminant ID65. This assumption limits the material properties in the scene to grey scale. The $n v R_{D}$ model outputs a smoothed delayed version of the input light signal simulated at the occupant's eye level, accounting for shifts in spectral sensitivity, the effect of intermittent patterns, different exposure durations, and adaptations to prior light history. The application of the $n v R_{D}$ model thus does not support a point-in-time evaluation, as the current response depends on past inputs.

The outcome of this model is evaluated over a 24-hour day and gives a cumulative response $\left(\mathrm{R}_{\mathrm{D}}\right)$. The cumulative response $R_{D}$ is mainly sensitive to total light intensity and duration of light exposure, therefore providing a measure of daily light dose, which is independent of circadian timing. At any time during the day, it is possible to return an intermediate value if, for example, it is relevant to evaluate a spatial position for shorter periods of time. This is especially relevant in buildings, where our occupation may span anywhere from a couple of minutes to several hours or days. Setting minimum targets or performance goals is 
necessary to evaluate non-visual health potential as we would like to know how much daylight we need, within a given space, from a given position and view, to achieve desired health potential. Given a reference profile of an ideal light exposure, the $n v R_{D}$ model can produce a target for evaluating performance. The $n v R_{D}$ model is described in detail in [16].

\subsection{Visualization of Results}

The $\mathrm{mSC}$ and $\mathrm{nvR}_{\mathrm{d}}$ performance modules take luminance and/or illuminance data as input for a series of view positions within a geometry model and present the results in a format that illustrates whether performance thresholds have been met across each view direction (Section 4.5) over an established time series. The production of simultaneously spatial and temporal data helps to inform designers, engineers, and building operators about where and when daylight might affect human perceptual and nonvisual responses in the built environment. While a preliminary (static) visualization of results for both $\mathrm{mSC}$ and $n v R_{D}$ is provided for a select architectural case study in this paper, future development of this platform will allow users to interact with results across view positions and over time. An overall picture of average daily performance can be just as useful as a detailed assessment of hourly performance or an instantaneous prediction across view directions.

\section{SELECTION OF ARCHITECTURAL CASE STUDY}

For the demonstration of this simulation-based workflow, we selected an architectural case study that presents a multitude of interior daylight conditions due to its patterned glass façade, varied interior layout, and mix of programmatic uses. The Ryerson Student Learning Center (SLC) in Toronto, designed by Snøhetta and Zeidler Partnership (opened to the public in 2012), is surrounded by a dense urban context, ranging in height from 3 to 9 stories (Figure 2).

Considered as a programmatic expansion to the neighboring library, the SLC houses student work/study spaces and staff offices - both open and enclosed. The daylight design concept harnesses natural illumination using high ceilings and open spaces with translucent frit to minimize direct sunlight and diffuse daylight deep within the interior spaces. The selection of a large fritted pattern on the exterior glass façade produces strong visual effects under direct sunlight.

\subsection{Selection of Spaces for Comparative Study}

The Ryerson SLC contains 9 floors, but to demonstrate the impacts of architectural form, orientation, and façade patterns on human-centric daylight performance, we decided to simulate a series of points across the 6 th and 7 th floors, which provide two plan configurations. The 6th floor is composed of informal open study space across a series of ramped floor levels. Using a 5-meter spacing, the floor plan was divided into a grid of 59 points, offset a minimum of 2 meters from the façade and circulation cores.

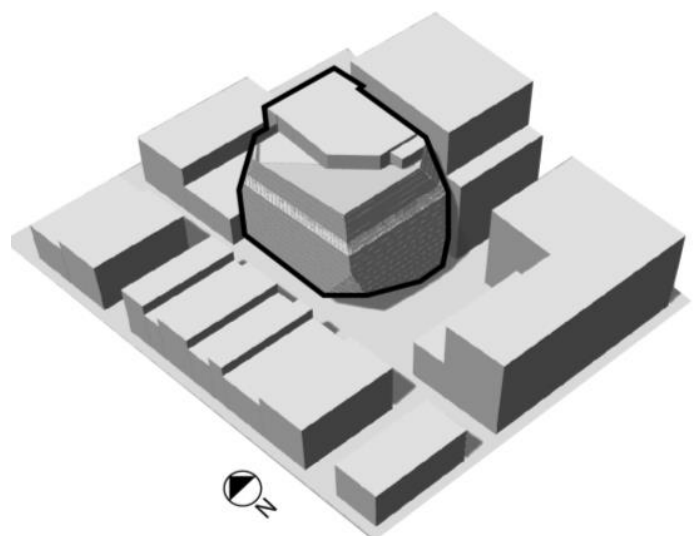

Figure 2. The Ryerson SLC is shown in its surrounding urban context as a digital rhino model.

A 5-meter grid was used to analyze a moderate number of interior view position, while limiting computational expense. Figure 3 shows the layout of space, distribution of sensor points, and a sample of rendered view directions from a select view position. The 7 th floor is split into three open study spaces, flanked by two rows of enclosed study rooms, as seen in Figure 3. All enclosed study rooms look out onto the open study spaces through an interior glazed wall. While many of these spaces require electric light at all times of the day due to insufficient natural illumination deep within the floor plate, we decided to simulate only the daylight to evaluate the carbon-neutral potential for interest and health-related lighting performance. Using the same spacing as for the $6^{\text {th }}$ floor, with the addition of points at the center of each enclosed study room, we established a grid of 76 points.

\subsection{Translation of Geometry Model into RAD Format}

The geometry of our selected case study was received as a DXF and imported into Rhinoceros. After re-grouping layers by material definition, we re-built the glass façade so frit patterns were individual glass objects and could properly read material transmission values. We defined a 5 $\mathrm{m}$ grid of points at eye level $(1.21 \mathrm{~m}$ from the floor while sitting) and exported those point locations as a text file. Depending on the specific nature of a desired analysis, a denser array of view positions could be used, but this would result in higher computational cost and must be considered alongside other input selections.

Using the DIVA toolbar, we exported our model and material definitions in the RAD format and imported them into the platform. We selected 28 semi-annual instances ( 7 hourly instances on each of January 13, February 28, April 14, and May 30) to give us a comprehensive snap shot of a symmetrical half year. This time series was developed from the Lightsolve method [19], which uses 56 symmetrical instances to interpolate an annual temporal profile of illuminance-based data. We chose 8 view directions per view position (135 points between the $6^{\text {th }}$ and $7^{\text {th }}$ floors) to conduct a series of illuminance and luminance-based simulations as inputs for our $\mathrm{mSC}$ and $\mathrm{nvR}_{\mathrm{D}}$ models. 

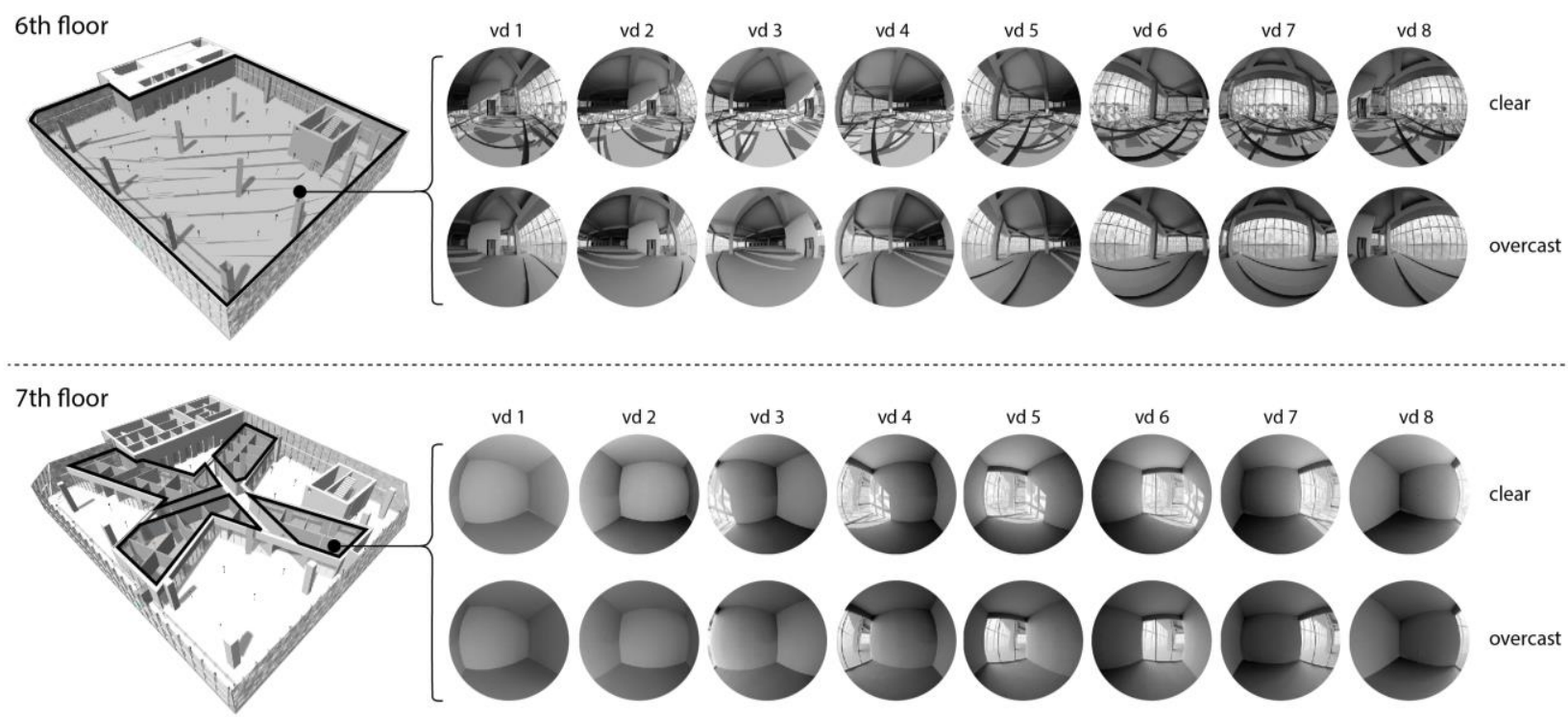

Figure 3. This image shows the two floors $\left(6^{\text {th }}\right.$ above and $7^{\text {th }}$ below) selected for analysis in our case study, alongside a sample of hemispherical renderings produced from each selected view position and view direction (1-8).

\subsection{Interpretation of Performance Modules}

For each view position and view direction $(60,480$ in total $=$ 135 view positions $\mathrm{x} 8$ view directions $\mathrm{x} 28$ instances $\mathrm{x} 2$ sky conditions) we applied the mSC algorithm to rendered hemispherical images [8]. For the same view positions and directions, the cumulative response $R_{D}$ was also calculated using effective irradiance [18].

\section{Visual Interest}

Two threshold values were used to categorize results of the $\mathrm{mSC}$ algorithm into three categories:

1. Calming, $\mathrm{mSC}<6.96$

2. Neutral, $\mathrm{mSC}<6.96,<11.75$

3. Exciting, $\mathrm{mSC}>11.75$

Results in this paper are presented as both the average daily mSC achieved across each view direction, but also as instantaneous moments. The average daily $\mathrm{mSC}$ allows the user to visualize a compact overview of the perceptual impact on each view direction, while the instantaneous hourly results allow him/her or see how those results vary over time. Results for each view direction are shown using colored arrows, with the length determined by the output of the mSC model and the color (cyan, grey, and magenta) determined by the thresholds listed above.

\section{Non-visual Health Potential}

A threshold value $T=4.2$ [16] was used to categorize the resulting daily cumulative responses $R_{D}$ into four categories:

1. Not achieved during the day, $\mathrm{R}_{\mathrm{D}}<\mathrm{T}$ (poor)

2. $\quad$ Achieved during the day, $\mathrm{R}_{\mathrm{D}}>\mathrm{T}$ (fair)

3. Achieved am or pm, $\mathrm{R}_{\mathrm{D}}>\mathrm{T}$ (good)

4. Achieved both am and pm, $R_{\mathrm{D}}>\mathrm{T}$ (excellent)
Achieving $R_{D}>T$ over the period of full day is not necessarily considered sufficient. The goal of $\mathrm{T}=4.2 \mathrm{can}$ be achieved during mornings or afternoons only if the duration of the solar day is sufficient, since the $n v R_{D}$ model depends on duration. By binning the results into am and pm the user can better understand the influence of time of day and how it can affect the accumulation of dose received. The binning of the data should be adjusted to every case study depending on specific program use. Results are shown with colored arrows in each view direction, with length indicating the magnitude of $\mathrm{R}_{\mathrm{D}}$ and the color indicating the threshold it falls into (dark green, light green, yellow, or orange). The desired performance is to achieve $\mathrm{R}_{\mathrm{D}}>\mathrm{T}$ before noon (am) or/and after noon (pm).

\section{RESULTS}

The results of our exemplary analysis are show at both building scale and occupant scale. Figure 4 shows two axonometric views of the $7^{\text {th }}$ floor with average daily $\mathrm{mSC}$ and daily dose predictions for $n v R_{D}$ on April 14 under clear skies. Figure 5 shows a zoom in on one view position on the $7^{\text {th }}$ floor (in a closed private study room) with daily average and instantaneous predictions of $\mathrm{mSC}$ across the day. Figure 6 shows average daily $\mathrm{mSC}$ and daily dose predictions for nvR $R_{D}$ on February 28 for both the $6^{\text {th }}$ and $7^{\text {th }}$ floors under clear and overcast sky conditions overlaid in plan. Figure 6 also shows a frequency distribution of model predictions for each floor under clear and overcast sky conditions for each of the 4 days included in our analysis.

\subsection{Visual Interest}

If we look at the results for February 28, the $6^{\text {th }}$ floor shows mostly exciting predictions for average daily $\mathrm{mSC}$ under clear sky conditions (Figure 6a), with a slight shift toward more neutral predictions under overcast skies (Figure 6b). 
The $7^{\text {th }}$ floor shows a significant shift towards calming predictions under both sky conditions (Figures 6e \& 6f), with the peripheral open-study spaces achieving much more exciting predictions than the closed study rooms on the interior.

While we can draw certain spatial conclusions from the overall daily averages in Figures 4 and 6, some of which are fairly intuitive, Figure 5 shows a more nuanced overview of the dynamic hourly experience within a single view position. In this instance (April 14, clear sky), the prediction for excitement shift dramatically across each view direction depending on the time of day and resulting sun position. The instantaneous predictions in Figure 5 illustrate the variable nature of perceptual performance as shifting sun positions from one moment to the next can change the evaluation of excitement in our visual field. Depending on the intended program use and qualitative

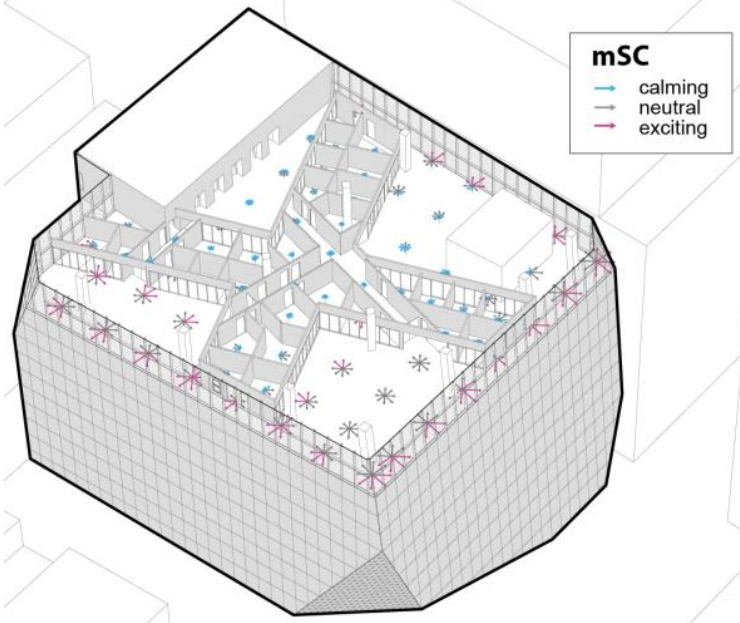

ambiance in an architectural space, these hourly performance predictions can be useful for the designer to know when, over the course of the day and year, impacts of daylight are likely to alter an occupant's emotional state.

\subsection{Health Potential}

Figure $6 \mathrm{c}-6 \mathrm{~h}$ shows the daily cumulative response $\mathrm{R}_{\mathrm{D}}$ across each viewpoint in plan on February 28. The results for the $6^{\text {th }}$ floor show excellent performance throughout the space under clear sky (Figure 6c). Lower light intensities caused by overcast sky conditions show reduced performance in view directions facing North and East, where viewpoints did not exceed the desired performance (Figure 6d). During solar hours for each of the 4 days the percentage frequency of the 4 performance categories (Section 3.4) is counted for all viewpoints in the scene and displayed using a stacked bar graph.

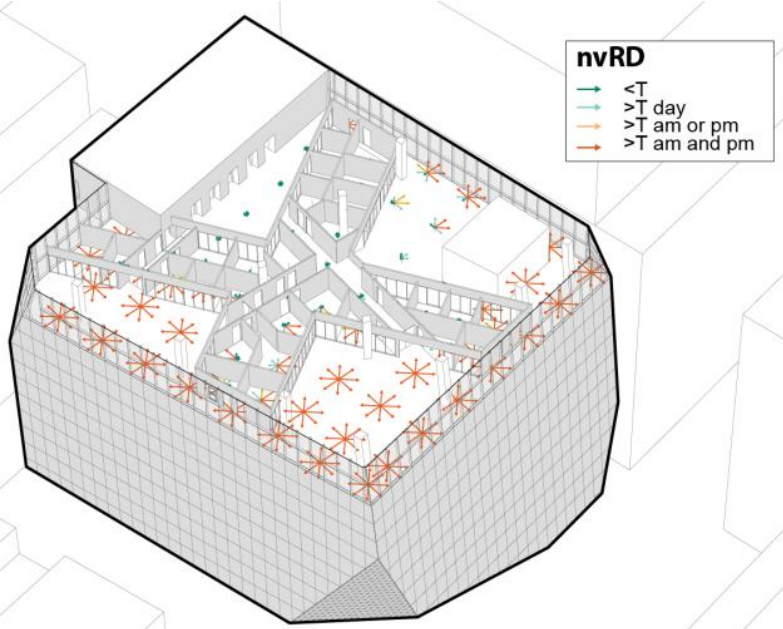

Figure 4. Showing two axonometric views with average daily $\mathrm{mSC}$ and cumulative response $\mathrm{R}_{\mathrm{D}}$ for each point in the $7^{\text {th }}$ floor on April 14 under clear sky conditions. The length of arrow is determined by the output of each model and colored by threshold (described in Section 3.4).

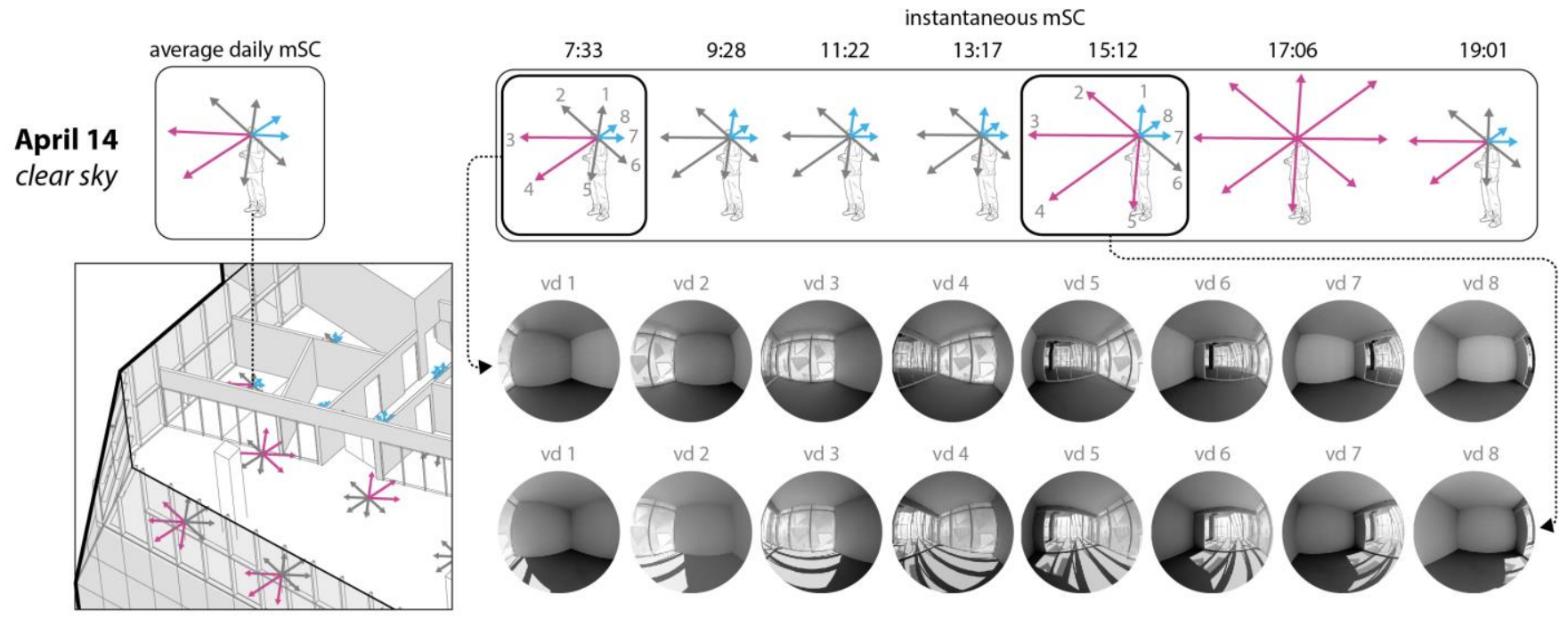

Figure 5. Shows a zoom in on one view position on the $7^{\text {th }}$ floor. Average daily predictions of $\mathrm{mSC}$ are shown on the left (April 14 under clear skies), with instantaneous hourly predictions of $\mathrm{mSC}$ shown on the right, with associated hemispherical renderings for two instances (7:33 and 15:12) to illustrate relationships between occupant prediction and rendered daylight conditions. 


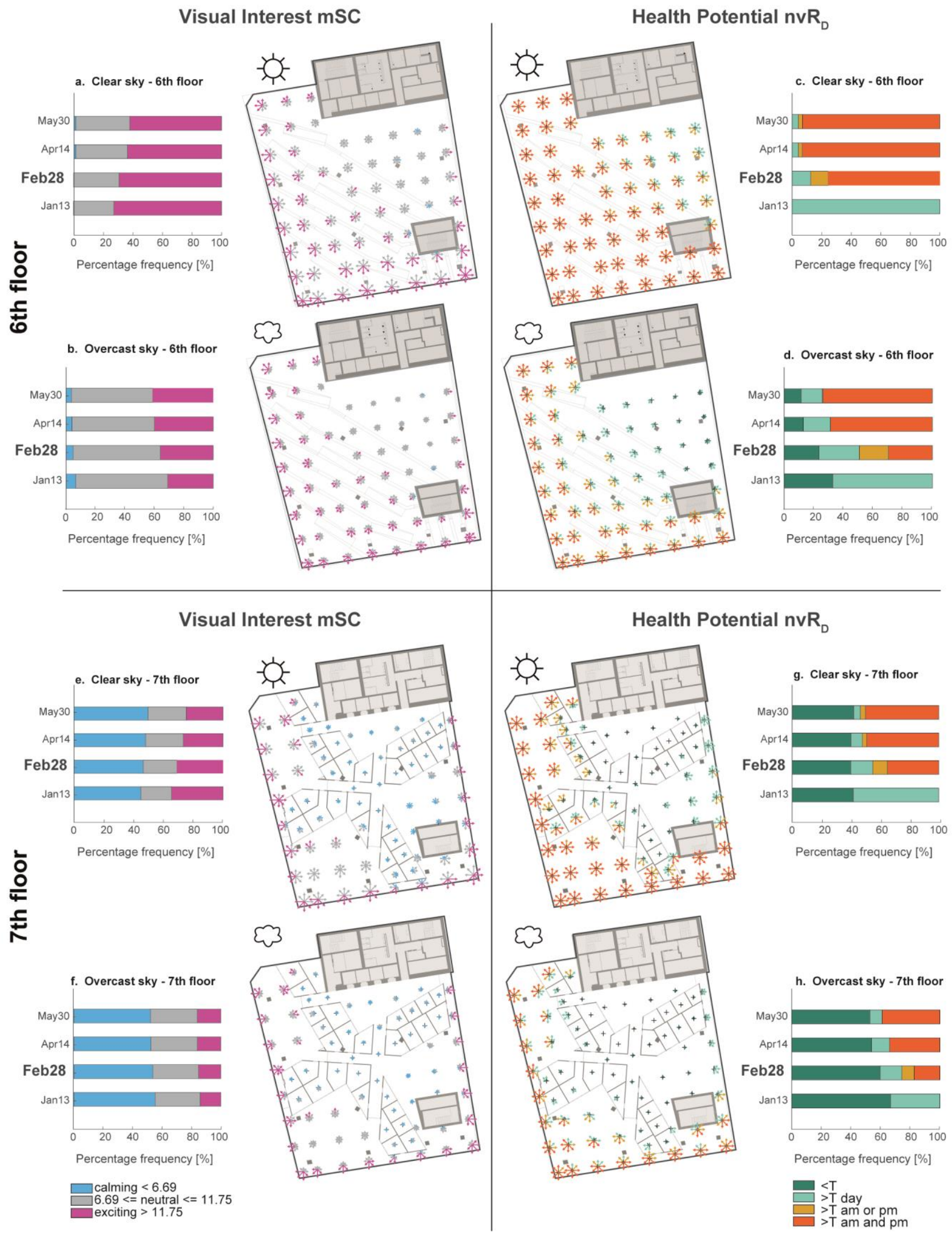

Figure 6. Average daily values for $\mathrm{mSC}$ (on the left) and cumulative daily dose predictions for $\mathrm{nvR}_{\mathrm{D}}$ (on the right) are overlaid in plan to show the impact of clear vs. overcast skies on each view position and view direction in the $6^{\text {th }}$ and $7^{\text {th }}$ floors. 
The results for the 6th floor under clear sky conditions are similar for most days of the year except during the darkest winter months, where the number of solar hours limits the overall health potential. As expected, the performance under overcast skies is reduced. Interestingly, the category of achieving $\mathrm{T}=4.2$ during am or pm increases on February 28, which means that achieving non-visual health potential is more sensitive to timing of occupation for overcast than clear skies around this time of year. This can be explored in more details by analyzing different time periods of the day.

The results for the 7th floor are less spatially homogeneous as compared to the 6th floor, which is explained by the partition of the $7^{\text {th }}$ floor into open and enclosed study rooms. Less than half of the simulated points belong to open study spaces (30/76) resulting in a lower overall performance for the $7^{\text {th }}$ floor. Under clear sky conditions on February 28 good and excellent performance is achieved for $45 \%$ of the viewpoints (Figure 6g), which reduces to $22 \%$ under overcast conditions (Figure 6h). As seen in Figures $6 \mathrm{~g}$ and 6h, enclosed study rooms receive much less light than open study spaces. Under overcast sky conditions almost none of the viewpoints in enclosed spaces (ca. 1\%) achieve the desired performance, while it is achieved under clear sky conditions in the enclosed rooms facing South and West.

\section{CONCLUSION}

This paper has introduced a simulation platform to evaluate in parallel two performance modules in daylight perception and health potential. Developed to automate the process of assessing human-centric performance predictions across a range of view positions and view directions within a given geometry model, the proposed platform creates a user-friendly support for daylight simulation. While past studies have used a similar workflow on a single view position in space, this paper offers a more streamlined protocol, allowing for the analysis of many view positions in a computationally efficient way. The speed of simulations is significantly improved through shared ambient files and data storage is optimized by keeping only the illuminance and luminance data necessary for the analysis of the performance modules. Visualizations of the data presented in this paper, while preliminary, provide insights regarding the daylighting performance of visual interest and non-visual health potential at the scale of the occupant, but also at the scale of the building. An array of view positions allows the user to compare performance predictions across multiple points in the case study and understand the impact of architectural composition on those predictions.

\section{ACKNOWLEDGEMENTS}

This work was supported by the Ecole Polytechnique Fédérale de Lausanne (EPFL) and through grants from the Swiss National Science Foundation [grant number
153018] and the Velux Stiftung Foundation [grant number 936].

\section{REFERENCES}

1. Rockcastle S, Andersen M. Measuring the dynamics of contrast and daylight variability in architecture: A proof-of-concept methodology. Building and Environment, 81 (2014).

2. Wymelenberg $\mathrm{K}$, Inanici $\mathrm{M}$. The effect of luminance distribution patterns on occupant preference in a daylit office environment, Leukos 7(2) (2010).

3. Veitch J, Newsham G. Preferred luminous conditions in open plan offices: Research amd practice recommendations. Light. Res. Technol. 32(4) 2000.

4. Loe D, Mansfield K, Rowlands E. Appearance of lit environment and its relevance in lighting design: Experimental study Light. Res. Technol., 26 (3) (1994).

5. Cetegen D, Veitch J, Newsham G. View size and office illuminance effects on employee satisfaction. Ljubljana, Slovenia : Proceedings of Balkan Light (2008).

6. Tiller D, Veitch J. Perceived room brightness: Pilot study on the effect of luminance distribution, Light. Res. Technol., 27(2) (1995).

7. Parpairi K, Baker N, Steemers K, Compagnon R. The luminance differences index: a new indicator of user preferences in daylit spaces. Light. Res. Technol., 34(1) (2002).

8. Rockcastle S, Amundadottir ML, Andersen M. Contrast measures for predicting perceptual effects of daylight in architectural renderings. Light. Res. Technol., (online first) (2016).

9. Hubalek S, Brink M, Schierz C. Office workers' daily exposure to light and its influence on sleep quality and mood. Light. Res. Technol., 42(1) (2010).

10. Smolders KC, de Kort YA, van den Berg S. Bright light and mental fatigue: Effects on alertness, vitality, performance and physiological arousal. J. Environ. Psychol., 39 (2014).

11. Smolders KC, de Kort YA, van den Berg SM. Daytime light exposure and feelings of vitality: Results of a field study during regular weekdays. Environ. Psychol, 36 (2013).

12. Zeitzer JM, Dijk DJ, Kronauer RE, Brown EN, Czeisler CA. Sensitivity of the human circadian pacemaker to nocturnal light: melatonin phase resetting and suppression. J. Physiol., 3 (2000).

13. Berson DM, Dunn FA, Takao M. Phototransduction by Retinal Ganglion Cells That Set the Circadian Clock. Science, 295(5557) (2002).

14. Hattar S, Liao HW, Takao M, Berson DM, Yau K. Melanopsincontaining retinal ganglion cells: architecture, projections, and intrinsic photosensitivity. Science, 295(5557) (2002).

15. Amundadottir ML. Light-driven model for identifying indicators of non-visual health potential in the built environment. Ecole Polytechnique Fédérale de Lausanne, 2016.

16. Amundadottir ML, Rockcastle S, Sarey Khanie M, Anderden M. A human-centric approach to assess daylight in buildings for non-visual health potential, visual interest and gaze behavior. Building and Environment, (online first) (2016).

17. Rizzi A, Algeri G, Medeghini D, Marini A. A proposal for contrast measure in digital images. Aachen, Germany: Second European Conference on Color in Graphics, Imaging and Vision (2004).

18. Amundadottir,ML, Lockley SW, Andersen M. Unified framework to evaluate non-visual spectral effectiveness of light for human health. Light. Res. Technol. (online first), (2016).

19. Kleindienst S., Bodart M., Andersen M. Graphical Representation of Climate-Based Daylight Performance to Support Architectural Design. Leukos, 5(1) (2008). 